\title{
Sistemas de Informação Governamental para Monitoramento e Avaliação das Políticas Públicas: quadro de desafios e perspectivas de avanços
}

Luciano Pezza Cintrão José Luis Bizelli²

1 Especialista em Gestão Pública de Cidades e Docente em curso sobre Sistemas Integrados de Informação da Escola Nacional de Administração Pública (ENAP) desde 2008. Coordenou o Programa de Governança para a Administração Municipal do Governo do Estado de São Paulo (http://www.governancamunicipal.sp.gov.br/), dedicado à criação de um ambiente de gestão da informação compartilhada entre o Estado e o Município, por meio da utilização de recursos tecnológicos e qualificação dos servidores municipais.

2 Professor Livre Docente em Gestão de Políticas Públicas da Faculdade de Ciências e Letras da Universidade Estadual Paulista "Júlio de Mesquita Filho" (UNESP), Campus de Araraquara. Foi Diretor da Faculdade de Ciências e Letras da UNESP Araraquara no quadriênio 2009-2012. 


\section{Resumo}

ORGANIZAÇÕES GOVERNAMENTAIS TÊM COMO DESAFIO PARA SUA GOVERNANÇA A NECESSIDADE DE APRIMORAR SEUS SISTEMAS DE MONITORAMENTO E AVALIAÇÃo OUE MENSUREM $O$ DESEMPENHO DE SUAS INTERVENÇÕES E DISPONIBILIZE PARA A SOCIEDADE INFORMAÇÕES SOBRE OS RESULTADOS ALCANÇADOS. $O$ TEXTO ANALISA OS DESAFIOS E AVANÇOS DOS INSTRUMENTOS DE MONITORAMENTO E AVALIAÇÃO PARA PRODUZIR CONHECIMENTOS OUE POSSAM SER INSERIDOS NO CONTEXTO DE TOMADA DE DECISÃO DOS AGENTES PÚBLICOS. PARA TANTO AS INFORMAÇÕES TÊM OQUE SER CONFIÁVEIS, ATUALIZADAS E COMPLETAS, CRIANDO VETORES DE ACELERAÇÃO PARA A MODERNIZAÇÃO DA CAPACIDADE INFORMACIONAL DO ESTADO COMO UM TODO.

\section{Abstract}

GOVERNMENT ORGANIZATIONS HAS AS CHALLENGE FOR THEIR GOVERNANCE THE REQUIREMENT TO IMPROVE EVALUATION SYSTEMS THAT MEASURE THE ACTING OF THEIR MEDIATIONS AND PROFFER FOR SOCIETY INFORMATIONS ABOUT ACHIEVEMENTS. THE TEXT ANALYZE THE CHALLENGES AND ADVANCES OF EVALUATION TOOLS FOR PRODUCE GUIDELINES THAT COULD BE INSERTED AT CONTEXT OF DECISION-MAKING FROM PUBLIC AGENTS. THEREUNTO INFORMATIONS MUST BE RELIABLE AND REAL-TIME, CREATING VECTORS OF HASTE FOR MODERNIZATION OF INFORMATIONAL CAPACITY OF STATE AS A WHOLE.

\section{PALAVRAS-CHAVE:}

Gestão Pública; Sistemas de Informação; Controle; Avaliação; Monitoramento; Tomada de Decisão. 


\section{Introdução}

Nos últimos anos, os aspectos relativos à acessibilidade digital e ao controle social sobre fluxos informacionais importantes vêm se incorporando às práticas democráticas. A sociedade - permeada por diferentes sistemas de informação e comunicação - tem se qualificado, por meio da democratização da informação, melhorando sua capacidade para avaliar e julgar as ações dos governos e de seus representantes.

Nesse contexto, as organizações governamentais incorporam ao quadro de desafio da governança ${ }^{3}$ a necessidade de aprimorar seus sistemas de avaliação $^{4}$ a fim de mensurar 0 desempenho de suas intervenções e disponibilizar para a sociedade informações sobre os resultados alcançados.

Como instrumento de gestão do setor público, os sistemas de avaliação necessitam produzir conhecimentos que possam ser inseridos no contexto de tomada de decisão. Surge, assim, o desafio de disponibilizar informações confiáveis, atualizadas e tempestivas para lastrear o processo de tomada de decisão, e, com ele, a necessidade de modernização da capacidade informacional do Estado como um todo.

No Brasil, a modernização da capacidade informacional do Estado encontra limites na cultura organizacional de diversos setores da Administração Pública, nos quais imperam os procedimentos que, na maioria das vezes, reproduzem ainda o modelo burocrático que atende à excessiva verticalização de estruturas decisórias, hierarquizadas e centralizadas, incapazes de atender às demandas de Gestão por Resultados, de acordo com um modelo gerencial proposto pelo Plano Diretor da Reforma do Estado 5 .

Essa realidade traz para o quadro de desafios a necessidade de incorporar sistemas de monitoramento e avaliação (M\&A) às práticas administrativas das instituições governamentais, como meios indispensáveis para fornecer informações precisas, atualizadas e em tempo real para a elaboração de políticas públicas cidadãs, para o monitoramento de sua implantação e para a avaliação de resultados obtidos a partir do financiamento público.

\section{Os Sistemas de Monitoramento e Avaliação}

A institucionalização dos sistemas de M\&A vem ganhando força no Brasil à medida que respondem a demandas, por um lado, de transparência e efetividade do gasto público pela sociedade, seja pelo fortalecimento das instituições democráticas, seja pela pressão da arrecadação tributária, e de outro, daquelas decorrentes da estruturação de um conjunto mais amplo de políticas sociais, instituídas como direitos na Constituição de 1988. Esta institucionalização também responde à profissionalização crescente da Administração Pública nas três esferas de governo e à introdução de sistemáticas mais sofisticadas de planejamento e controle na gestão das políticas públicas ${ }^{6}$.

O processo de institucionalização dos sistemas de M\&A exigiu do Estado brasileiro a mo- 
dernização do aparato normativo de garantia ao direito dos cidadãos de acesso facilitado às informações ${ }^{7}$ primárias, íntegras, autênticas e atualizadas, mantidas ou custodiadas por órgãos e entidades públicas, reconhecendo este direito como condição básica e fundamental da democracia.

Nesse sentido, o País firmou tratados internacionais, em especial a Parceria para Governo Aberto - celebrada entre o Brasil e sete outros países em setembro de 2011 - e inseriu no arcabouço jurídico da legislação pátria em especial o Decreto $s / n^{\circ}$ de 15 de setembro de 2011, que institui o Plano de Ação Nacional sobre Governo Aberto e pela Lei $n^{\circ}$ 12.527, de 18 de novembro de 2011 (Lei de
Acesso à Informação - LAl) - normativas que regulam o acesso a informações previsto no inciso XXXIII do artigo 50, no inciso II do § 30 do artigo 37 e no $§ 20$ do artigo 216 da Constituição Federal.

No entanto, para que as premissas dos princípios de $\underline{\text { dados abertos }}^{8}$ sejam definitivamente incorporadas às rotinas das instituições públicas, há necessidade de um esforço modernizante capaz de alterar antigas práticas arraigadas na cultura dos órgãos públicos relacionadas à manutenção de cadastros desatualizados, procedimentos de coleta que não garantem a integridade e autenticidade do $\underline{\text { dado }}^{9}$, ausência de rotinas de atualização, sistemas informatizados fechados, bases de

3 Governança significa a capacidade financeira e administrativa do Estado de implantar decisões tomadas nas arenas decisórias governativas. A essa definição acrescentamos o sentido de percepção de ganho efetivo que o cidadão tem com relação às políticas públicas implantadas.

4 Avaliação de programas: processo gerador de aprendizagem que, por meio do uso de procedimentos sistemáticos de pesquisa, verifica os resultados e a efetividade dos programas na solução do problema que justificou sua criação (Melo, 2012).

5 BRASIL, 1995.

6 GUIMARÃES E JANNUZZI (2004).

7 Informação: conjunto de dados organizados de tal forma que tenham valor ou significado em algum contexto (Fonte: (Secretaria de Logística e Tecnologia da Informação. Instrução Normativa No 4, 12 de abril de 2012. Art. $2^{\circ}$ ).

8 Dados abertos: dados públicos representados em meio digital, estruturados em formato aberto, processáveis por máquina, referenciados na rede mundial de computadores e disponibilizados sob licença aberta que permita sua livre utilização, consumo ou cruzamento (Fonte: Secretaria de Logística e Tecnologia da Informação. Instrução Normativa nº 4, 12 de abril de 2012. Art. $2^{\circ}$ ).

9 Dado: sequência de símbolos ou valores representados em algum meio e produzidos como resultado de um processo natural ou artificial (Fonte: Secretaria de Logística e Tecnologia da Informação. Instrução Normativa n 4, 12 de abril de 2012. Art. $\left.2^{\circ}\right)$.

Revista Brasileira de Monitoramento e Avaliação | Número 5 | Janeiro-Junho de 2013 
dados fragmentadas, entre outras, que resultam na indisponibilidade de informação qualificada para uso no planejamento da ação governamental e no monitoramento e avaliação de políticas públicas, tornando o dado disponível impróprio para fins de constituição de uma arquitetura de dados abertos e não útil para informar a sociedade.

Sendo assim, torna-se imprescindível e premente a adoção de uma nova Arquitetura para os Sistemas de Informação Governamental (ASI-g), a fim de que os legítimos anseios sociais por um governo mais eficiente na gestão de seus negócios e efetivo na implantação de suas ações sejam alcançados.

Nesse novo modelo de ASI-g, o foco volta-se para o cidadão, privilegiando o conceito de formato aberto ${ }^{10}$, de informação como um ativo de governo, de reuso da informação e de acesso simplificado dos usuários às informações por meio de meios eletrônicos, com especial atenção à transparência da gestão fiscal, às informações orçamentárias e às prestações de contas.

\section{A Inadequação dos Sistemas de Informação Governamenta|l11}

A Arquitetura dos Sistemas de Informação de Governo (ASI-g) tem princípios articulados no formato clássico de hierarquização dos órgãos públicos, conforme modelo burocráti$\mathrm{CO}^{12}$. As burocracias têm sua fonte de legitimidade no poder racional-legal - cuja finalidade é alcançar meios eficientes para atingir fins, nos limites da lei - ou seja, as organizações públicas são sistemas racionais, apresentando características como formalidade, impessoalidade e profissionalismo na condução do trabalho.

Com o tempo, as distorções características da racionalidade instrumental refletiram-se na ASI-g, dentre as quais a formalização excessiva, o legalismo exacerbado e o corporativismo acentuado, resultando em sistemas que não apoiam adequadamente o processo decisório, já que foram concebidos para dar apoio às decisões administrativas, não dando suporte às decisões políticas. Ademais, tal arquitetura tem resultado em sistemas de informação fragmentados, sem padrões de troca de informações, com abordagem centralizada, não incorporando necessidades setoriais.

Consequentemente, a ASI-g resultante não fornece um modelo e uma metodologia de aquisição e utilização estruturada da informação para as arenas decisórias. Algumas deficiências, portanto, ficam evidentes na ASI-g, como a inexistência de dados atualizados e confiáveis. A aquisição de dados hoje cria para os usuários um retrabalho: os dados são coletados e armazenados, mas não estão disponíveis quando necessário. Além disso, há dificuldade concreta de acesso a dados. A ausência de normas criteriosas de acesso e de regras para obtenção de informações nas bases públicas cria barreiras para seu uso, tanto por atores públicos quanto pelo cidadão em geral.

Outrossim, a mudança de concepção do Estado e os próprios avanços da democracia passaram a influenciar o processo de toma- 
da de decisões, envolvendo múltiplos atores organizados muitas vezes em processo de participação mais direto e difuso, como o orçamento participativo.

Nesse cenário, os problemas decorrentes da obsolescência dos sistemas de informações governamentais como um todo e dos sistemas estruturantes em especial se avolumaram e os gestores - cada dia mais sozinhos e sufocados com os incêndios do dia a dia passaram a demandar informações para decisões que fossem além de ações tópicas sobre os sintomas visíveis e mais compatíveis com as novas responsabilidades que lhes foram atribuídas.

Para compreender o quadro de desafios envolvidos na institucionalização dos sistemas de M\&A, é preciso conhecer o variado rol de sistemas de apoio à decisão em uso no aparelho governamental, que vai desde sistemas que servem para a programação econômica até os utilizados como instrumentos de gestão, ou seja, aqueles que permitem, por exemplo, a apuração de responsabilidades sobre uma decisão tomada, a autorização para a ação e o conhecimento da situação de cada projeto e atividade em execução, etc.

As informações produzidas estão intrinsecamente relacionadas aos sistemas de contabilidade e orçamento públicos, já que o orçamento é, na prática, a ferramenta de trabalho que permite a ligação entre os sistemas de planejamento e as finanças. Por meio do orçamento, são monetarizados os projetos e atividades previstos nos planos de ação, permitindo que os gestores equacionem o futuro, executando realisticamente os recursos financeiros disponíveis.

Assim, as informações sobre políticas e programas - aquelas que possibilitam o controle gerencial - advêm do orçamento, especialmente quando se apresentam aliadas a sistemas que atribuem expressão quantitativa física e financeira para a mensuração das ações governamentais realizadas. Enquanto o orçamento serve ao planejamento e gerenciamento governamental, as informações para tomada de decisão, controle e avaliação de desempenho advêm da contabilidade. Por isso, no Brasil, os sistemas gerenciais de in-

10 Formato aberto: formato de arquivo não proprietário, cuja especificação esteja documentada publicamente e seja de livre conhecimento e implementação, livre de patentes ou qualquer outra restrição legal quanto à sua utilização (Fonte: Secretaria de Logística e Tecnologia da Informação. Instrução Normativa n 4, 12 de abril de 2012. Art. 20).

11 As reflexões contidas neste tópico foram suscitadas pelo trabalho de Antônio de Pádua Ferreira Passos \& Priscila de Souza Cavalcante Castro (PASSOS \& CASTRO, 2009).

12 WEBER,2004.

Revista Brasileira de Monitoramento e Avaliação | Número 5 | Janeiro-Junho de 2013 
formação para o planejamento e controle de políticas governamentais estão baseados nas técnicas de orçamento e contabilidade previstas na Lei n 4.320/64.

As principais ferramentas para a gestão e a administração do Governo Federal são denominadas sistemas estruturadores e estão sendo operados pelo Serviço Federal de Processamento de Dados (SERPRO). Esses complexos sistemas, executados em mainframe e largamente utilizados pelo setor público, armazenam informações vitais para o funcionamento do aparelho estatal em diferentes processos (Quadro 1).

\section{- QUADRO 1: PROCESSOS TÍPICOS DOS SISTEMAS ESTRUTURADORES}

- Elaboração e acompanhamento do orçamento;

- Administração de recursos humanos;

- Alocação de cargos;

- Compras governamentais;

- Acompanhamento dos programas e ações do governo;

- Administração financeira e contábil; e

- Patrimônio da União.

A abordagem centralizada dos sistemas estruturadores sem incorporação das necessidades dos órgãos setoriais resulta na proliferação de subsistemas setoriais com baixa integração com os primeiros. De fato, os Sis- temas de Informação (SI) atualmente em uso no Governo Federal não foram desenhados segundo uma concepção de interoperabilidade, a qual viesse a privilegiar a ligação do governo como o ambiente externo. Para solucionar os problemas relativos à agregação de informações entre os diferentes sistemas, o Governo Federal desenvolveu o projeto I3_ gov, que tem como objetivo central criar as condições para a construção de um sistema gerencial a partir da integração com os sistemas estruturadores.

\section{Arranjos Institucionais para Modernização dos Sls}

O Governo Federal incumbiu a Secretaria de Logística e Tecnologia da Informação (SLTI) de articular com outros órgãos de governo e conduzir as políticas públicas para a área de TI. A SLTI opera a função de Secretaria Executiva do Comitê Executivo de Governo Eletrônico, órgão responsável pela normatização da contratação e do uso das Tecnologias de Informação e Comunicação (TIC) no Governo Federal. Estão na competência da SLTI as atividades de Fomento; Posições internacionais (Governança da Internet e Fóruns de padronização) e Projetos estratégicos (Banda Larga nas Escolas; Portal do Software Público; Integração dos Sistemas Estruturadores). 
Os padrões e metodologias para modernização dos Sls para a gestão estão baseados em dois instrumentos: o I3-GOV (Integração, Inteligência e Informações de Governo), que contempla quatro Sls agregadores de informações produzidas pelos sistemas estruturadores (Quadro 2); e o e-PING (Padrões de Interoperabilidade de Governo Eletrônico), que define a arquitetura da Integração, Inteligência e Informações de Governo.

Os padrões fixados pelo 13-GOV objetivam estabelecer critérios para responder às demandas por informações cada vez mais complexas para apoio à decisão. Nesse sentido, os sistemas gerenciais devem disponibilizar informação, de forma ágil e eficiente, para que as arenas decisórias do governo possam controlar e avaliar o desempenho dos programas de trabalho em curso. O conceito subjacente ao I3-GOV é que recursos de informação do governo constituem valiosos ativos econômicos. Ao garantir que a informação governamental possa ser rapidamente localizada e transmitida entre os setores público e privado, mantidas as obrigações de privacidade e segurança, o governo auxilia no aproveitamento máximo dos ativos, impulsionando e estimulando a economia do País.
As políticas traçadas pelo I3-GOV estão fundamentadas nos seguintes princípios:

- Governos de todo o mundo estão investindo fortemente no desenvolvimento de políticas, processos e estabelecimento de padrões em TIC, montando estruturas dedicadas para obter a interoperabilidade e buscando o provimento de serviços de melhor qualidade a custos reduzidos;

- A existência de uma infraestrutura de TIC que se preste como alicerce para a criação dos serviços de governo eletrônico é o pré-requisito para o fornecimento de melhores serviços à sociedade, a custos mais baixos;

- Um governo moderno e integrado exige sistemas igualmente modernos e integrados, interoperáveis, trabalhando de forma íntegra, segura e coerente em todo o setor público; e

- Políticas e especificações claramente definidas para interoperabilidade e gerenciamento de informações são fundamentais para propiciar a conexão do governo tanto no âmbito interno como no contato com a sociedade e, em maior nível de abrangência, com o resto do mundo - outros governos e empresas atuantes no mercado mundial. 
A e-PING - que estabelece os Padrões de Interoperabilidade de Governo Eletrônico - é concebida como uma estrutura básica para a estratégia de governo eletrônico, aplicada inicialmente ao Governo Federal - Poder Executivo. Permite racionalizar investimentos em TIC por meio do compartilhamento, reuso e intercâmbio de recursos tecnológicos. A arquitetura e-PING define um conjunto mínimo de premissas, políticas e especificações téc- nicas que regulamentam a utilização das TIC no Governo Federal, estabelecendo as condições de interação com os demais poderes, esferas de governo e com a sociedade em geral. É coordenada pela Secretaria de Logística e Tecnologia da Informação do Ministério do Planejamento (SLTI/MP); Instituto Nacional de Tecnologia da Informação, da Presidência da República (ITI/PR) e SERPRO, empresa pública do Ministério da Fazenda.

\section{- OUADRO 2: SISTEMAS AGREGADORES DE INFORMAÇÕES OUE FORMAM O I3-GOV}

- $\quad$ Sistema de Execução de Despesas Operacionais: fornecimento de informações agregadas a partir de dados de despesa armazenados no SIAPE (Sistema Integrado de Administração de Pessoas) e SIASG (Sistema Integrado de Administração de Serviços Gerais);

- Sistema de Administração de Despesas: fornecimento de informações agregadas a partir de dados de planejamento e execução de despesas armazenadas no SIAFI (Sistema Integrado de Administração Financeira), comparativamente aos dados de despesa armazenados no SIAPE e SIASG;

- $\quad$ Sistema de Informações Agregadas de Despesa: fornecimento de informações agregadas a partir de dados armazenados nos dois protótipos anteriores e de dados de despesa armazenados nos demais sistemas estruturadores, SIORG (Sistema de Informações Organizacionais), SIDOR (Sistema Integrado de Dados Orçamentários), SIEST (Sistema Integrado das Empresas Estatais) e SIGPLAN (Sistema de Informações Gerenciais de Planejamento). Considerar sistemas do âmbito do Ministério do Planejamento que controlam receitas, como é o caso do SIAPA (Sistema Integrado de Administração Patrimonial).

- Sistema de Comunicação (broker) e Web Services. 


\section{Considerações Finais}

Os reclamos sociais por respostas à questão do gasto público e sua relação com o desenvolvimento da sociedade trouxeram para o quadro de desafios das instituições governamentais a necessidade de aprimorar seus sistemas informacionais a fim de disponibilizar informações úteis para lastrear o processo decisório relativo à estruturação de políticas públicas - que vão se tornando cada vez mais analíticas, para mensuração do desempenho de suas intervenções - por meio de sistemas de monitoramento e avaliação para disponibilizar à sociedade informações sobre os resultados alcançados, em atenção aos princípios da transparência governativa.

Gerar governança através da incorporação e difusão de Tecnologias de Informação e Comunicação tornou-se um compromisso moral dos governos, mercê da introdução no ordenamento pátrio da legislação de transparência governativa e ética dos administradores, relacionada à formação de cidadãos com condições de fiscalizar as arenas públicas e suas ações.
A arquitetura dos sistemas de informação do governo precisou colocar-se a serviço de uma maior governabilidade e de um planejamento estratégico de políticas atrelado aos princípios de accountability ${ }^{13}$, dentro da perspectiva de que o controle eficiente sobre os resultados das políticas públicas e a determinação daqueles entes que são responsáveis pela ação administrativa geram incremento positivo na prestação de serviços da máquina administrativa ao cidadão.

O conceito de accountability traz a ideia de que duas condições são indispensáveis para fiscalizar competentemente o Estado: pelo lado da sociedade, a ampliação da consciência cidadã em torno de reivindicações cujo atendimento pelo poder público proporcione melhoria das condições de vida de toda a coletividade; e pelo lado do Estado, o provimento de informações completas, claras e relevantes para a população, expandido assim o número de controladores e reforçando - pela prestação de contas direta ao titular do poder,

13 O conceito de accountability tem duas conotações básicas da accountability política: a) a capacidade de resposta dos governos (answerability), isto é, a obrigação dos oficiais públicos informarem e justificarem seus atos; e b) a capacidade (enforcement) das agências de accountability (accounting agencies) de impor sanções e perda de poder para aqueles que descumpriram os deveres públicos. A noção de accountability é, basicamente, bidimensional: envolve capacidade de resposta e capacidade de punição (answerability e enforcement)(Schedler, 1999).

Revista Brasileira de Monitoramento e Avaliação | Número 5 | Janeiro-Junho de 2013 
o povo - a legitimidade das políticas públicas e a segurança de seus atos.

A introdução e o desenvolvimento desses sistemas de M\&A no processo de governança pública pressupõem, por um lado, que os subsistemas setoriais implantados internamente na máquina administrativa sejam abertos e com possibilidades de interatividade e, por outro lado, que na comunidade sejam desenvolvidas habilidades que permitam a absorção e utilização de forma crítica e cidadã dessas informações, transformadas em um processo de gestão do conhecimento.

Dentro dos avanços da democracia, a difusão dos sistemas de M\&A apresenta-se como esperança de conquista de uma nova cidadania, à medida que favorece a luta por melhoria dos serviços públicos que atendam às demandas das populações. Para tanto, os dados sobre o orçamento público devem ser transparentes e constituírem-se em base para o planejamento estratégico setorial e intersetorial.

É importante que os gestores públicos tomem a dianteira do processo, quando não interferindo para facilitar e dar respostas técnicas aos governos, pelo menos ajudando a compreender as mudanças provocadas ${ }^{14}$. Tudo isso exige uma postura administrativa diferenciada e um esforço dos governos para introduzir mudanças que deem novas capacidades ao administrador público para que ele assuma o seu papel mais político na sociedade brasileira. 


\section{Referências bibliográficas}

BIZELLI, J. L.; CINTRÃO, LUCIANO P. Inovação, Governança Pública e Desenvolvimento Local. In: CASAGRANDE, E. E. (Org.). Intervenção fiscal e suas dimensões: teoria, política e governança. São Paulo: Cultura Acadêmica, 2012. p. 225-247.

BRASIL. Câmara da Reforma do Estado. Plano Diretor da Reforma do Aparelho do Estado. Brasília, 1995. Disponível em: <http://www.bresserpereira.org.br/Documents/MARE/PlanoDiretor/ planodiretor.pdf>. Acesso em: 20 nov. 2011.

GUIMARÃES, J. R. S.; JANNUZZI, P. M. IDH, indicadores sintéticos e suas aplicações em políticas públicas: uma análise crítica. In: ENCONTRO NACIONALDE ESTUDOS POPULACIONAIS, 14. Anais... Caxambu, 2004.

MELO, Patrícia Coimbra Souza. Monitoramento e avaliação na administração pública federal [manuscrito]: os desafios do PPA 2012/2015. 2012. Disponível em: <www.abrasil.gov.br/publicações>.
PASSOS, Antônio de Pádua Ferreira; CASTRO, Priscila de Souza Cavalcante. O Orçamento e a Dívida Pública Federal. In.: SILVA, Anderson Caputo; CARVALHO, Lena Oliveira de; MEDEIROS, Otavio Ladeira de (Org.). Dívida Pública: a experiência brasileira. Brasília: Secretaria do Tesouro Nacional, 2009.

SCHEDLER, Andreas. Conceptualizing accountability. In: SCHEDLER, A.; DIAMOND, L.; PLATTNER, M. F. (Eds.). The self-restraing state: power and accountability in new democracies. Boulder and London: Lynne Rienner Publishers, 1999.

WEBER, Max. Economia e sociedade: fundamentos da sociologia compreensiva. Regis Barbosa e Karen Elsabe Barbosa (Trad.). v. 2. Brasília-DF: Editora da Universidade de Brasília, 2004.

Revista Brasileira de Monitoramento e Avaliação | Número 5 | Janeiro-Junho de 2013

Sistemas de Informação Governamental para Monitoramento e Avaliação das Políticas Públicas: quadro de desafios e perspectivas de avanços 